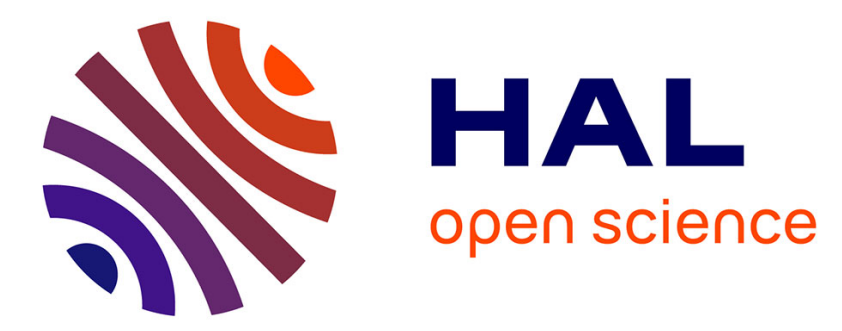

\title{
Low frequency locally resonant metamaterials containing composite inclusions
}

\author{
Guy Bonnet, Vincent Monchiet
}

\section{To cite this version:}

Guy Bonnet, Vincent Monchiet. Low frequency locally resonant metamaterials containing composite inclusions. Journal of the Acoustical Society of America, 2015, 137, pp.3263. 10.1121/1.4921273 . hal-01165845

\section{HAL Id: hal-01165845 \\ https://hal.science/hal-01165845}

Submitted on 14 Mar 2016

HAL is a multi-disciplinary open access archive for the deposit and dissemination of scientific research documents, whether they are published or not. The documents may come from teaching and research institutions in France or abroad, or from public or private research centers.
L'archive ouverte pluridisciplinaire HAL, est destinée au dépôt et à la diffusion de documents scientifiques de niveau recherche, publiés ou non, émanant des établissements d'enseignement et de recherche français ou étrangers, des laboratoires publics ou privés. 
Low frequency locally resonant metamaterials containing composite inclusions

\author{
Guy Bonnet a) and Vincent Monchiet \\ Université Paris-Est \\ Laboratoire Modélisation et Simulation Multi-Echelle, MSME UMR 8208 CNRS \\ 5 boulevard Descartes, 77454 Marne la Vallée, France
}

a) e-mail: guy.bonnet@univ-paris-est.fr 


\begin{abstract}
One main feature of metametarials is the occurrence of a negative dynamic mass density that is produced when an inner local resonance is present. The inner resonance can be obtained in composite materials containing composite inclusions. For suitable ratios of the physical properties of the constituting materials, the composite inclusions act as springmass systems. The scaling of physical properties leading to such an inner resonance and the associated effective dynamic properties of materials containing composite inclusions are recalled briefly. The resonance frequencies and dynamic mass densities are obtained in a closed form for materials containing cylindrical composite fibers or spherical composite inclusions, after solving the related boundary value elasticity problems.
\end{abstract}




\section{INTRODUCTION}

A significant amount of research has been devoted recently to metamaterials, firstly in the field of electromagnetism and more recently in the field of acoustics. This field of research can be traced back to 1968 Veselago's paper ${ }^{36}$ on electromagnetism, as explained in Shamonina and Solymar ${ }^{32}$. For acoustic materials, an experimental evidence for acoustic band gaps in two-dimensional composites with fibers was obtained by Vasseur et al. ${ }^{35}$. From a fundamental point of view, it appears that interesting properties of acoustic metamaterials come from resonance, either due to the collective behaviour of inclusions or to the presence of local resonators ${ }^{22 ; 23}$. This last case corresponds to the occurence of inner resonance . Numerous papers in this field were published that either study the properties of metamaterials from a theoretical approach ${ }^{1 ; 15 ; 16 ; 18 ; 8}$ or display experimentally the properties of such materials ${ }^{19 ; 33 ; 26 ; 38 ; 29}$. An important aspect of these works is that the dynamic behaviour exhibits an "effective dynamic mass density", that becomes negative at certain frequencies, as predicted or observed by several authors $2 ; 3 ; 11 ; 38 ; 19 ; 37$.

In the following, the study will be restricted to elastic composites made of composite inclusions. Even so, the amount of literature devoted to this field is impressive. A first method for studying this problem consists of providing the elastodynamic solution for waves propagating through a periodic composite by decomposition into Bloch waves $^{17 ; 27 ; 10 ; 28}$. However, the solutions depend essentially on the ratios of physical 
properties of the components of the composite (elastic parameters and mass densities). Therefore, a strong physical insight can be gained using an asymptotic series expansion of the dynamic solution for different assumptions on the ratios between physical parameters: elastic coefficients and mass densities. First results showing by such a method the occurrence of bandgaps in elastic composites were obtained in early works ${ }^{2 ; 3}$, but with the development of the ideas on metamaterials, new results have been obtained more recently with the use of asymptotic expansions in dynamic elasticity ${ }^{30 ; 5 ; 6 ; 4 ; 34}$. More specifically, Auriault and Boutin ${ }^{4}$ have extended recently the method of asymptotic expansion to materials containing composite inclusions. They have shown that, under suitable assumptions, these composite inclusions act as inner resonators, leading to relatively lower values of frequencies characterizing the inner resonance. These results are the basis of the present paper. It is worthwhile noting that, compared with results coming from other publications in this field, an important aspect does appear in the papers written by Auriault and al. that are at the basis of our work ${ }^{2 ; 3 ; 4}$ : it is indeed shown in these papers that the use of different scalings of physical properties lead to different macroscopic behaviours. As a consequence, it is of prime importance to characterize the scaling properties leading to a specific dynamic behaviour.

In section 2, the results of the asymptotic expansion method in the case of materials containing composite inclusions are briefly recalled to point explicitly the set of physical 
parameters leading to inner resonance, the composite inclusions acting as "spring-mass resonators". In section 3, the inner motion of composite inclusions made of cylindrical fibers is studied; the eigenfrequencies and dynamic mass densities of these resonators are obtained in a closed form. The case of spherical composite inclusions is studied in section 4 and the full solution of the local elasticity problem is developed in this case, providing again the frequencies that are characteristic of the occurrence of negative mass densities.

\section{DYNAMIC BEHAVIOUR OF ELASTIC COMPOSITES CONTAINING RESONANT COMPOSITE INCLUSIONS}

Let us consider a composite material made of a matrix containing composite inclusions. These composite inclusions contain an inner inclusion (3) located inside a coating (2) and surrounded by the matrix (1) as shown in Fig.1

This composite material is defined by the geometry of the periodic cells containing the composite inclusions and by the physical properties of the constituent materials. All constituents are assumed elastic and isotropic. They are characterized by their mass densities $\rho^{(s)}$, their volume concentrations $c^{(s)}$ and their Lamé elastic parameters $\lambda^{(s)}, \mu^{(s)}$. $s$ corresponds to the matrix (1), the coating (2) or the inner inclusion (3).

In a recent paper, Auriault and Boutin ${ }^{4}$ have shown that, for chosen scaling parameters, a material containing composite inclusions can display a negative mass density due to the resonance of the composite inclusions acting as "spring-mass" resonators. In a 
Bonnet and Monchiet, JASA, p. 6

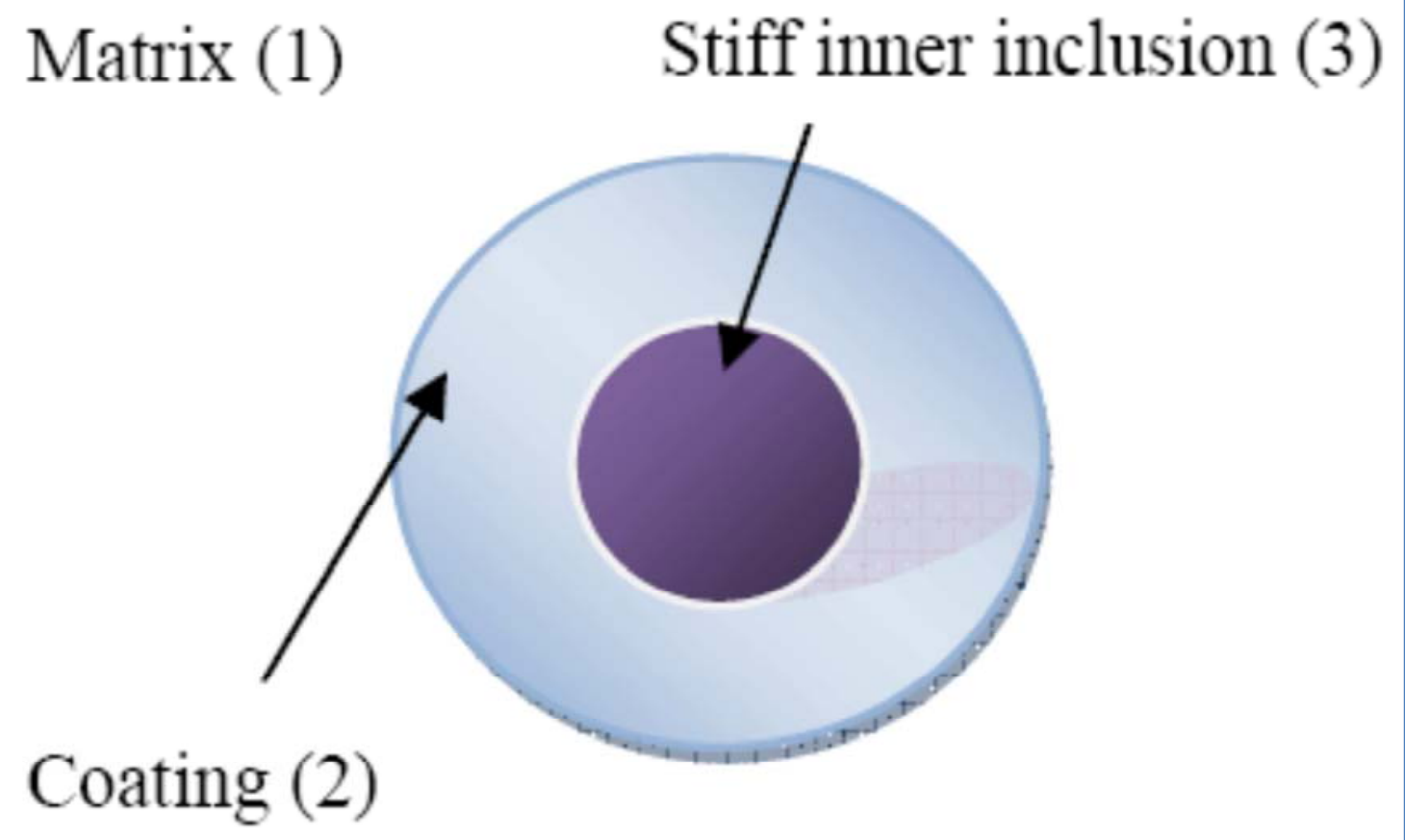

Figure 1: A composite inclusion within the matrix. 
first step, we define these scaling parameters. Next, we present the order of magnitude of the scaling parameters leading to inner resonance of "spring-mass" type and the dynamic mass density deduced from this kind of behaviour.

\section{A. Scaling parameters}

A first parameter is the scaling ratio that is defined by:

$$
\epsilon=\frac{l}{L}
$$

where $l$ is the size of the periodic cell and $L$ is the order of magnitude of the wavelength within the matrix in a chosen frequency range. The case of interest is when the size of the periodic cell is small compared with $L$ and $\epsilon<<1$.

Other scaling parameters contain the various physical parameters that characterize the constituents of the composite.

They comprise:

- the ratios between the different mass densities of the constituents $\frac{\rho^{(i)}}{\rho^{(j)}}$.

- the ratios between the orders of magnitude of the elastic coefficients of the different constituents. The order of magnitude of the elasticity coefficients of a given material will be defined by the value of $a^{(s)}=\lambda^{(s)}+2 \mu^{(s)}$ (that is a norm of the elasticity

tensor) . The scaling ratios related to elastic coefficients will be defined by $\frac{\lambda^{(i)}+2 \mu^{(i)}}{\lambda^{(j)}+2 \mu^{(j)}}=\frac{a^{(i)}}{a^{(j)}}$ : 


\section{B. Scaling assumptions leading to a spring-mass behaviour}

Using asymptotic expansion along the scaling parameter $\epsilon$, Auriault and Boutin ${ }^{4}$ studied the effect of different values of the previously defined scaling ratios on the overall behaviour of the composite. In the following, only one case will be considered, that leads to a dynamic behaviour characterizd by spring-mass resonance. It corresponds to these orders of magnitude of the scaling ratios:

$$
\begin{aligned}
& \frac{a^{(2)}}{a^{(1)}}=O\left(\epsilon^{2}\right) \\
& \frac{a^{(2)}}{a^{(3)}}=O\left(\epsilon^{p}\right), p \geq 1
\end{aligned}
$$

for the elastic parameters, and:

$$
\begin{aligned}
& \frac{\rho^{(2)}}{\rho^{(1)}}=O\left(\epsilon^{q}\right), q \geq 1 \\
& \frac{\rho^{(3)}}{\rho^{(1)}}=0(1)
\end{aligned}
$$

for the mass densities

It can be noticed that the scaling ratio for elastic parameters corresponds to a coating that is very soft compared with the matrix and the inner inclusion.

\section{Spring-mass behaviour}


Under the previously defined conditions, the composite has an effective behaviour for harmonic time excitation at radial frequency $\omega$ that is characterized by the dynamic equation

$$
\frac{\partial\left(a_{i j k l}^{(e f f)} \varepsilon_{k l}\right)}{\partial x_{j}}+\omega^{2} \rho_{i j}^{(e f f)} u_{j}=0
$$

where $a_{i j k l}^{(e f f)}$ are the "effective" elastic coefficients of the composite, $\varepsilon_{k l}$ are the components of the strain tensor, $u_{j}$ are the components of the displacement and $\rho_{i j}^{(e f f)}$ are the components of a frequency-dependent dynamic density. With a periodic array of composite inclusions, the effective elastic behaviour is not isotropic, contrarily to the behaviour of the constituents. Due to the scaling assumptions, the coating is very soft compared with the matrix. As a consequence, the effective elastic coefficients $a_{i j k l}^{(e f f)}$ can be computed as for a matrix that contains voids in place of composite inclusions.

Concerning the components $\rho_{i j}^{(e f f)}$ of the mass density, they must be considered as the ones of a tensor of second rank. In the following, it will be assumed that the composite inclusions have three orthogonal symmetry planes. In this case, the dynamic mass density is diagonal for a coordinate system whose axes are parallel to the symmetry planes, with:

$$
\rho_{i i}^{(e f f)}(\omega)=\langle\rho\rangle+c^{(3)} \rho^{(3)} \frac{\omega^{2}}{\omega_{i}^{2}-\omega^{2}}
$$

where Einstein's convention summation must not be applied to the repeated indice $i$ in 
$\rho_{i i}^{(e f f)},\langle\rho\rangle$ being the volume average of the density within the periodic cell and $\omega_{i}$ the resonance frequency of the composite inclusion given by:

$$
\omega_{i}^{2}=\frac{K_{i}}{m^{(3)}}
$$

$m^{(3)}$ is the mass of the inner inclusion and the stiffness $K_{i}$ is obtained by computing the reaction $F_{i}$ coming from the inner inclusion when this inner inclusion is subjected to a uniform displacement $w_{i}$ along axis $i$, the displacement over the outer boundary of the composite inclusion being null, with:

$$
F_{i}=-K_{i} w_{i}
$$

Due to the scaling and symmetry assumptions, it can be noticed that the inner inclusion behaves as a rigid solid compared with the coating and is subjected to a uniform translation. Under dynamic sollicitations, there is a dynamic displacement of the matrix and $w_{i}$ is the relative displacement $w_{i}=u_{i}^{(3)}-u_{i}^{(1)}$ of the inner inclusion with respect to the matrix.

It is worthwhile mentioning that the behaviour that is so described corresponds to a low frequency range. At higher frequencies, a more complicated dynamics involving the resonance of the coating itself must be taken into account and the previously defined dynamic mass density is no more appropriate. 
The objective of the paper is to compute the values of $K_{i}$ and $\rho_{i i}^{(e f f)}$ for two kinds of composite inclusions. These values are obtained by solving different boundary value elasticity problems in domain (2) corresponding to the coating.

\section{A physical approach to the dynamic density for an array of harmonic oscillators}

The physics leading to the previous expression of the dynamic density can be recovered by studying the behaviour of composite inclusions as harmonic oscillators contained within holes inside a very stiff matrix, as proposed by Milton and Willis ${ }^{23}$ and as shown in Fig. 2. This set is assumed to move with a displacement $u$ along $x_{1}$.

Let us consider the displacement $u^{(3)}$ of the mass of such an harmonic oscillator having a mass $m^{(3)}$ that is connected by a massless spring of stiffness $K$ to the matrix moving with the displacement component $u^{(1)}$. The mass of the matrix contained within a given unit cell is equal to $m^{(1)}$. The dynamic equation of the inner mass for a motion at radial frequency $\omega$ is given by:

$$
-K\left(u^{(3)}-u^{(1)}\right)=-m^{(3)} \omega^{2} u^{(3)}
$$

It leads to: 


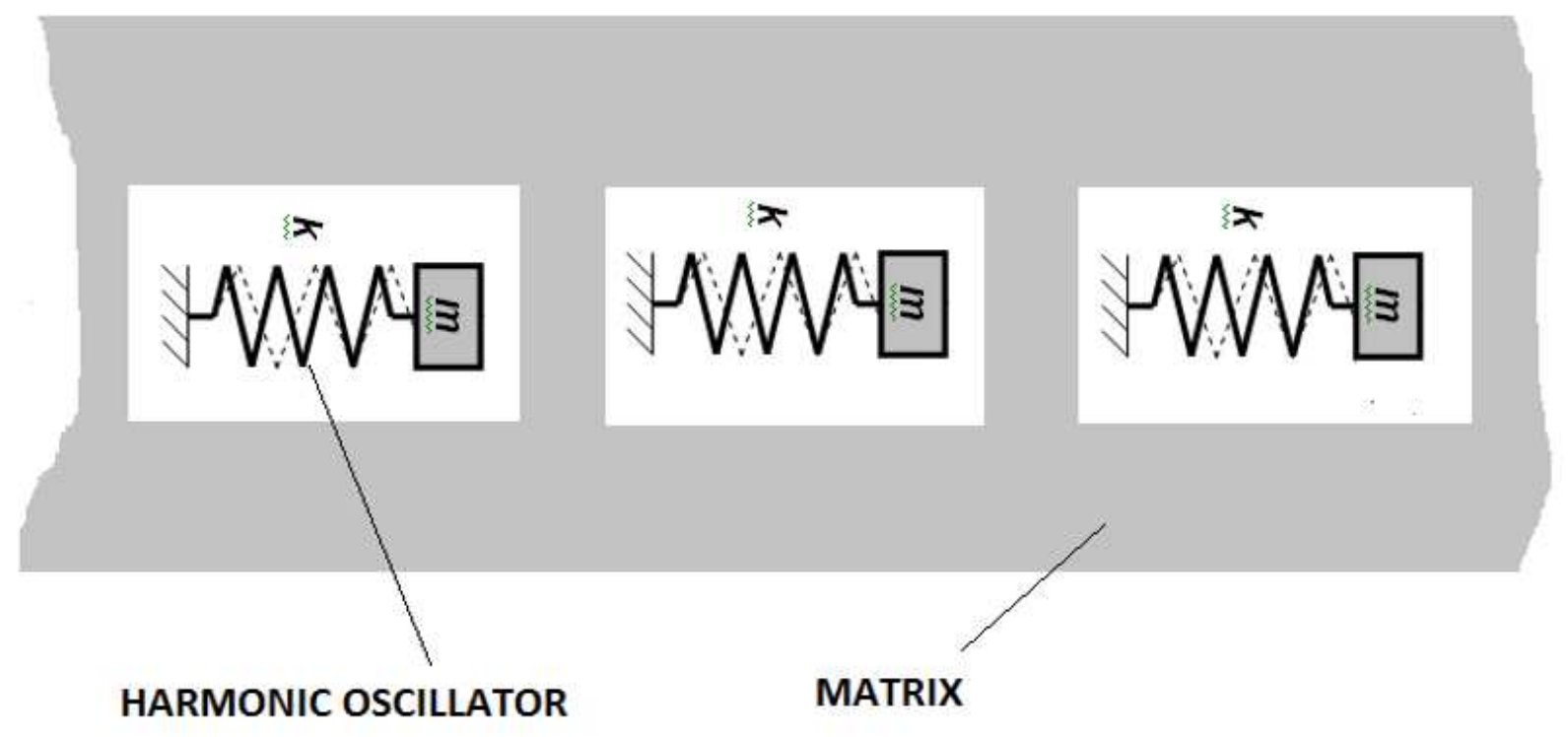

Figure 2: A periodic array of harmonic oscillators. 


$$
u^{(3)}=\frac{K}{K-m^{(3)} \omega^{2}} u^{(1)}=\frac{1}{1-\frac{\omega^{2}}{\omega_{r}^{2}}} u^{(1)}
$$

where $\omega_{r}=\sqrt{\frac{K}{m^{(3)}}}$ is the resonance frequency of the harmonic oscillator.

When a dynamic force $F$ is applied to a unit cell, one obtains:

$$
F=-\omega^{2}\left(m^{(1)} u^{(1)}+m^{(3)} u^{(3)}\right)
$$

Using the relationship between $u^{(3)}$ and $u^{(1)}$ and denoting the total mass within the period by $m^{t}, m^{t}=m^{(1)}+m^{(3)}$, one obtains:

$$
F=-\omega^{2} u^{(1)} m^{(e f f)}
$$

where

$$
m^{(e f f)}=m^{t}+m^{(3)} \frac{\omega^{2}}{\omega_{r}^{2}-\omega^{2}}
$$

Dividing this mass by the volume of the periodic cell leads to the expression of the dynamic mass density obtained previously. However, it is worthwhile mentioning that the result of homogenization contains in addition the scaling ratios leading to this kind of spring-mass system in 3D configurations and the boundary value problems to solve in order to obtain the stiffness coefficients. 
Bonnet and Monchiet, JASA, p. 14

\section{RESONANCE OF THE SPRING-MASS SYSTEM MADE OF COMPOSITE FIBERS}

Let us consider the case of a three-phase system containing composite fibers inside a matrix (Fig. 3). As explained in the previous section, the inner inclusion (radius $b$ ) located within the overall fiber (radius $a$ ) acts as a rigid body and the resonance frequency does not involve the properties of the matrix nor the elasticity of the inner inclusion.

The dynamic mass density is obtained from a dynamic problem related to a given overall displacement of the matrix. This one can be parallel to the direction of the fibers (i.e. longitudinal) or perpendicular to the direction of the fibers (i.e. transversal). Let us consider in a first step the case of a longitudinal motion.

\section{A. Longitudinal motion}

When the inertial forces due to the acceleration of the matrix are parallel to the direction of the fibers, the fibers move also parallelly to the same direction. The stiffness of the composite inclusion can be obtained by studying the inner fiber subjected to a displacement $W_{3}$ along the direction of the composite fiber, the matrix being fixed. It can be shown that the displacement within the coating is antiplane with respect to the plane $x_{1}, x_{2}$ perpendicular to the fibers. The relative displacement $\mathbf{w}$ inside the coating is oriented along $x_{3}$. Its component $w_{3}$ along this direction has a circular symmetry and is solution of: 


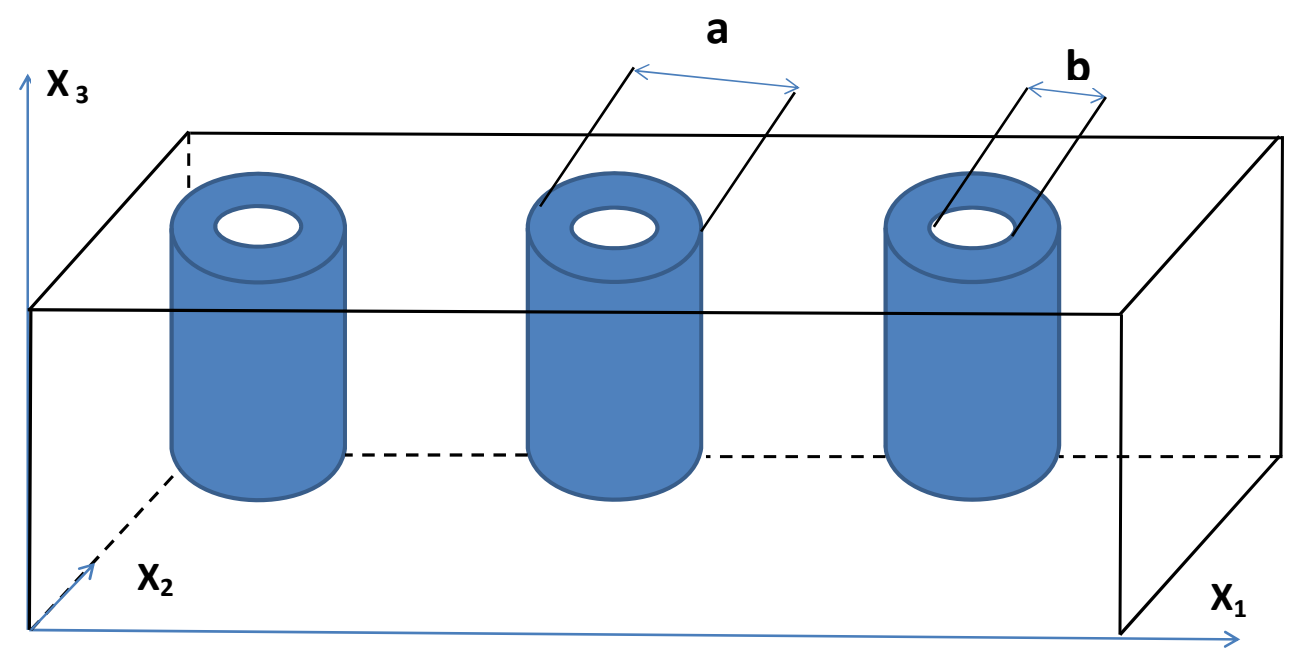

Figure 3: A composite comprising a matrix containing a periodic array of composite fibers made of a soft coating (radius a) surrounding a stiff inner fiber (radius b). 


$$
\frac{\partial^{2} w_{3}}{\partial x_{1}^{2}}+\frac{\partial^{2} w_{3}}{\partial x_{2}^{2}}=0
$$

The solution of this boundary problem is:

$$
w_{3}=W_{3} \frac{\ln \left(\frac{r}{a}\right)}{\ln \left(\frac{b}{a}\right)}
$$

leading to the shear strain $\tau$ along the inner fiber:

$$
\tau=\mu W_{3} \frac{1}{b \ln \left(\frac{b}{a}\right)}
$$

Integrating over the surface of the inner cylinder and dividing by the displacement $W_{3}$ of the inner fiber leads to the stiffness by unit length of fiber:

$$
K_{3}=2 \pi \mu \frac{1}{\ln \left(\frac{a}{b}\right)}
$$

The eigenfrequency $\omega_{r(3)}=\sqrt{\frac{K_{3}}{m^{(3)}}}$, where $m^{(3)}$ is the mass of inner fiber by unit length, is finally:

$$
\omega_{r(3)}=\sqrt{2 \frac{\mu}{\rho^{(3)}} \frac{1}{\ln \left(\frac{a}{b}\right) b^{2}}}
$$

This can be written as: 


$$
\omega_{r(3)}=\sqrt{\frac{\mu}{\rho^{(3)} a^{2}}} \omega_{d(3)}
$$

where the non-dimensional frequency $\omega_{d(3)}$ is a function of the ratio $\alpha=\frac{b}{a}$ between inner and outer radii of the composite fiber:

$$
\omega_{d(3)}=\sqrt{\frac{-2}{\ln (\alpha) \alpha^{2}}}
$$

This function presents only one minimum when $\alpha$ is between 0 and 1 . This minimum that is attained for $\alpha_{\text {min }}=\frac{1}{\sqrt{e}}$. For this value of $\alpha$, the nondimensional frequency is equal to $\omega_{d(3) \min }=2 \sqrt{e}$. This shows that, for a given outer radius of the composite fiber, a minimum is attained for this particular (named thereafter "optimal") geometry of the composite fiber.

\section{B. Transversal motion}

Due to the circular geometry of the section, the stiffness corresponding to the transversal motion does not depend on the direction of this motion in plane $x_{1} x_{2}$. Therefore, there is only one resonance frequency that is the same for all transversal motions.

The stiffness is obtained by enforcing a given transversal displacement $W_{1}$ of the inner inclusion, the external boundary of the coating being fixed. The reaction given by the 
coating makes it possible to obtain the eigenfrequency of the system.

The solution of such a problem rests on the complex variable solutions described in the classical Muskhelishvili's book ${ }^{25}$ for systems with circular boundaries. The application of this kind of solution to the case corresponding exactly to the previously described boundary value problem has been given by Gross ${ }^{12}$.

The components in cylindrical coordinates of the stress tensor produced by a given displacement $W_{1}$ of the inner cylinder are:

$$
\begin{aligned}
\sigma_{r r} & =\left[(3+\chi) \frac{A}{r}+2 A_{2} r+2 \frac{B_{2}}{r^{3}}\right] \cos \theta=B_{r} \cos \theta \\
\sigma_{\theta \theta} & =\left[(1-\chi) \frac{A}{r}+6 A_{2} r-2 \frac{B_{2}}{r^{3}}\right] \cos \theta \\
\sigma_{r \theta} & =\left[(1-\chi) \frac{A}{r}+2 A_{2} r+2 \frac{B_{2}}{r^{3}}\right] \sin \theta=B_{t} \sin \theta
\end{aligned}
$$

where $r$ is the radial coordinate, $\theta$ is the polar angle from $x_{1}$ axis , $\chi$ is a function of the Poisson's ratio $\nu$ by $\chi=3-4 \nu$ and the other constants are given by:

$$
\begin{aligned}
& A=-\frac{W_{1} \chi \mu\left(a^{2}+b^{2}\right)}{\chi^{2}\left(a^{2}+b^{2}\right) \ln \left(\frac{a}{b}\right)-\left(a^{2}-b^{2}\right)} \\
& A_{2}=\frac{A}{\chi\left(a^{2}+b^{2}\right)} \\
& B_{2}=-\frac{A a^{2} b^{2}}{a^{2}+b^{2}}
\end{aligned}
$$

The integration of the traction vector applied to the inner inclusion over the surface of the inner inclusion produces the linear density of force $F$ applied to the inner inclusion:

$$
F=\pi b\left(B_{r}-B_{i}\right)
$$


Then, the stiffness is given by $K_{1}=F / W_{1}=\pi b\left(B_{r}-B_{t}\right) / W_{1}$. Replacing $B_{r}$ and $B_{t}$ by their values leads to:

$$
K_{1}=\mu \frac{2 \pi \chi\left(\alpha^{2}+1\right)(1+\chi)}{\alpha^{2}-1-\chi^{2}\left(\alpha^{2}+1\right) \ln (\alpha)}
$$

This stiffness depends only on the elastic coefficients and on the geometry of the composite inclusion through the ratio $\alpha=b / a$.

Finally, the resonance frequency $\omega_{r(1)}$ for a displacement in plane $x_{1} x_{2}$ is given by:

$$
\omega_{r(1)}=\left[\frac{\mu}{\rho^{(3)} a^{2}}\right]^{1 / 2} \omega_{d(1)}(\chi, n)
$$

with:

$$
\omega_{d(1)}=\left\{\frac{2 \chi\left(\alpha^{2}+1\right)(1+\chi)}{\left[\left(\alpha^{2}-1-\chi^{2}\left(\alpha^{2}+1\right) \ln (\alpha)\right] \alpha^{2}\right.}\right\}^{1 / 2}
$$

The non-dimensional frequency $\omega_{d(1)}$ is only a function of the ratio $\alpha$ and of the Poisson's ratio (through $\chi$ ).

Studying the non dimensional frequency for different geometries, when $\alpha$ is varying from 0 to 1 , allows us again to show that $\omega_{d(1)}$ has always only one minimum $\omega_{d(1) \min }(\chi)$. This minimum is attained for $\alpha=\alpha_{\min }(\chi)$. It corresponds to an "optimal geometry" (i.e. the one giving the minimal eigenfrequency). The variation of $\alpha_{\min }$ as a function of the Poisson's ratio is given in Fig. 4. It can be seen that $\alpha_{\min }$ decreases with Poisson's ratio from $\alpha_{\min }=0.5$ for $\nu=0$ to $\alpha_{\min }=0.3$ for $\nu=0.5$. 


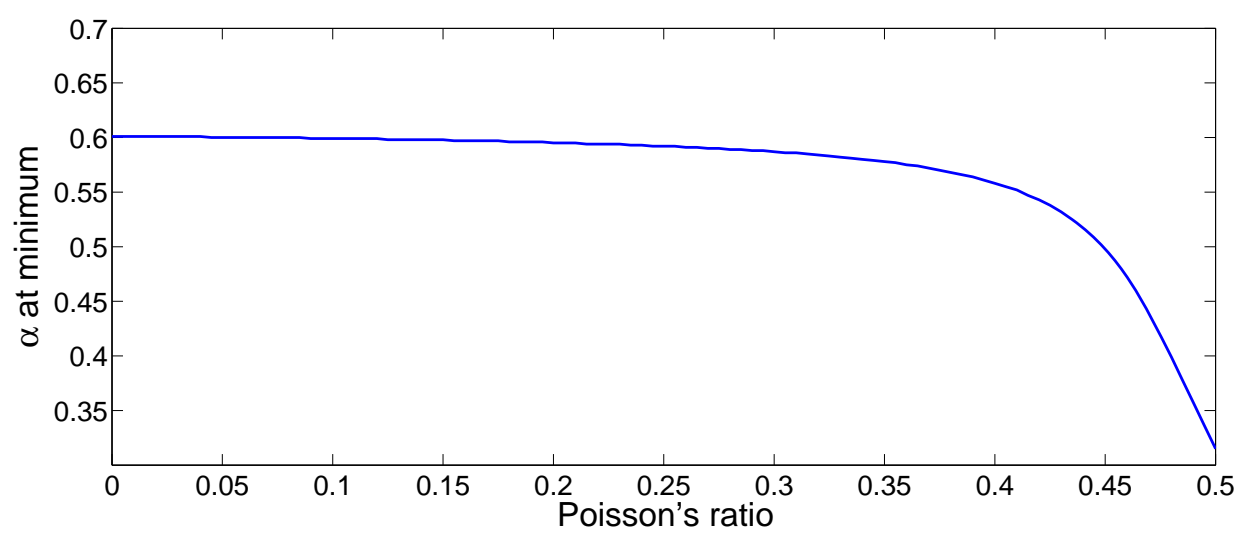

Figure 4: Ratio of the radii of the internal and external spheres at the minimum value of the resonance frequency as a function of the Poisson's ratio for a material containing composite fibers.

Then, Fig.5 presents the values of the non-dimensional resonance frequency $\omega_{d}=\omega_{d(1) \min }$ for the "optimal" geometry as a function of the Poisson's ratio. It can be seen that the non-dimensional frequency increases from $\omega_{d}=4$ for $\nu=0$ to $\omega_{d}=11$ at $\nu=0.5$.

These results make it possible to find easily the "optimal" geometry and the resonance frequency for given properties of the soft coating and of the inner inclusion.

As an example of application, let us now consider the case of a material containing composite cylindrical fibers made of a polyurethane foam $(\mathrm{E}=0.5 \mathrm{MPa}, \nu=0.3)$ containing steel wires $\left(\rho^{(3)}=7800 \mathrm{~kg} / \mathrm{m} 3\right)$. Fig. 6 shows the resonance frequency $(\mathrm{Hz})$ as a function of the outer radius of the composite fiber. The resonance frequency lies between $4 \mathrm{kHz}$ and 4 $\mathrm{MHz}$ for an external radius taken between $1 \mathrm{~mm}$ and $1 \mu \mathrm{m}$. 


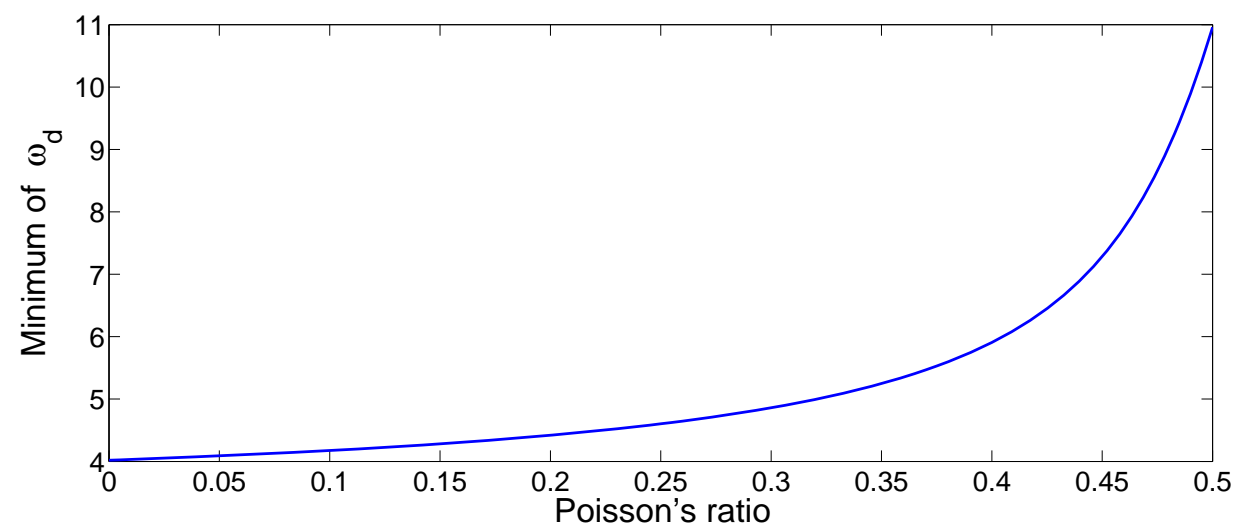

Figure 5: "Optimal" non-dimensional resonance radial frequency as a function of the Poisson's ratio for a material containing composite fibers.

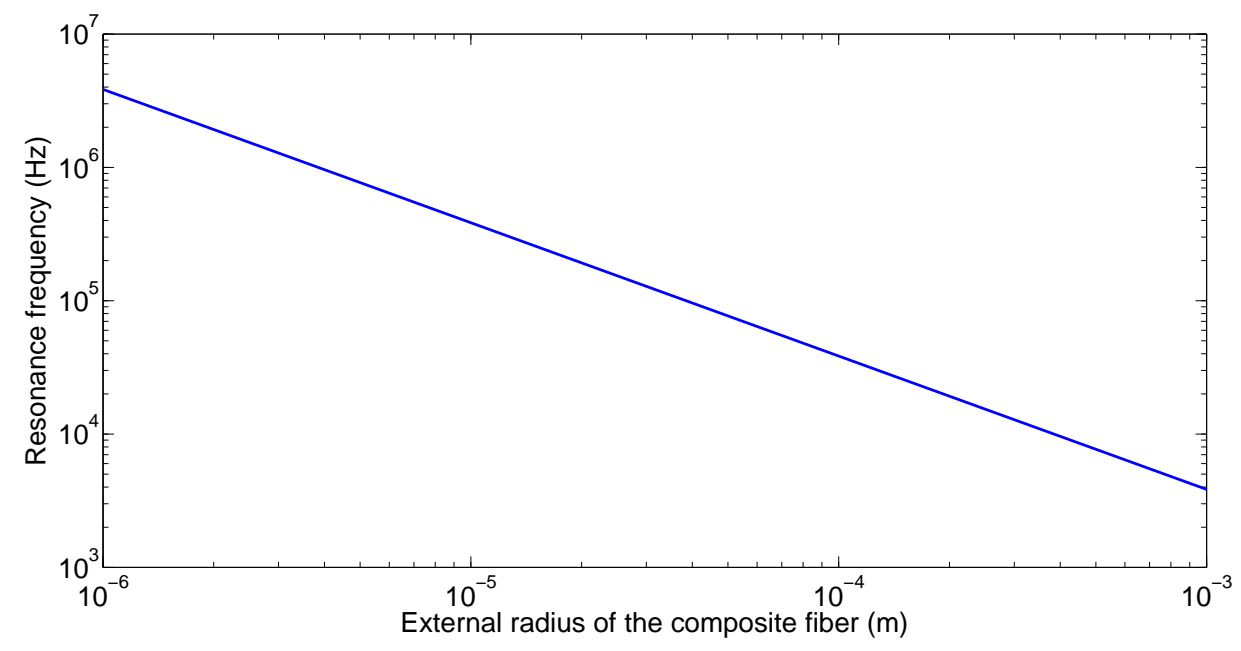

Figure 6: Resonance frequency as a function of the external radius (m) of the composite fibers for a material containing composite inclusions made of a polyurethane coating containing steel cylindrical wires. 
From the previous results, it comes that the dynamic mass density is given by the

second rank tensor whose non-null components are $\rho_{11}^{(e f f)}=\rho_{22}^{(e f f)}, \rho_{33}^{(e f f)} \neq \rho_{1}^{(e f f)}$. In this case, the dynamic density cannot be represented by a scalar but has a symmetry corresponding to the geometry of the composite fiber.

\section{RESONANCE OF THE SPRING-MASS SYSTEM MADE OF COMPOSITE SPHERES}

Let us now consider the case of inclusions made of composite spheres characterized by the internal radius $b$ and the external radius $a$, with notations similar to the ones of previous section for the properties of the coating and of the inner inclusion. As shown before, the stiffness of the composite inclusion is found by applying a uniform displacement on the inner inclusion, for a null displacement over the external surface of the coating. The solutions of elasticity theory for solids subjected to boundary conditions over spherical surfaces can be found in classical textbooks. Such solutions can be expressed using either Papkovich-Neuber solution ${ }^{21}$ or Love's scalar potentials ${ }^{20}$. One difficulty when using these solutions is to take into account the boundary conditions and an interesting method to write easily these boundary conditions has been introduced by Rahman and Michelitsch ${ }^{31}$. Using it, these authors found a few solutions for the full sphere or for spherical cavities within an infinite medium. This solution is adapted in the following to the case of a spherical layer subjected to boundary displacements on each spherical boundary. 


\section{A. The Love potentials}

The solution to the problem of displacement given on spheres is obtained in spherical coordinates $R, \theta, \varphi$ related to cartesian coordinates $x_{1}, x_{2}, x_{3}$ of the position vector $\mathbf{R}$ defined by:

$$
\begin{aligned}
& x_{1}=R \cos \theta \sin \varphi \\
& x_{2}=R \sin \theta \sin \varphi \\
& x_{3}=R \cos \varphi
\end{aligned}
$$

It was shown in the classical Love's book ${ }^{20}$ that a fully general displacement field that is solution of the isotropic elasticity problem is given by:

$$
\begin{aligned}
\mathbf{u}=\sum_{n=-\infty}^{\infty}[ & R^{2} \boldsymbol{\nabla} \psi_{n}-M_{n} \mathbf{R} \psi_{n} \\
& \left.+\nabla \phi_{n}+\nabla \times \boldsymbol{R} \chi_{n}\right]
\end{aligned}
$$

where $\psi_{n}, \phi_{n}, \chi_{n}$ are "solid" spherical harmonics, $\mathbf{R}$ is the vector position. $M_{n}$ are functions of $n$ and $\nu$ given by:

$$
M_{n}=2 \frac{2 \nu n+(1-2 \nu)(3 n+1)}{2 \nu(n+3)+(1-2 \nu)(n+5)}
$$

\section{B. Boundary conditions}

The hard point when using Love's solution is that the boundary conditions are given using the components of the surface displacement, but the displacement itself is expressed 
in terms of potentials. A simplifying method has been proposed by Rahman and Michelitsch ${ }^{31}$, based on results coming from Happel and Brenner ${ }^{14}$. These authors have shown that three scalar functions of the displacement along the surface can be computed when knowing only the surface displacement. Indeed, they have shown the following identities at the surface of a sphere of radius $a$ :

$$
\begin{array}{ll}
{\left[\mathbf{e}_{R} \cdot \mathbf{u}\right]_{R=a}} & =\mathbf{e}_{R} \cdot \mathbf{u}(a, \theta, \varphi) \\
{\left[\boldsymbol{R} \cdot \boldsymbol{\nabla} u_{R}-R \boldsymbol{\nabla} \cdot \boldsymbol{u}\right]_{R=a}} & =-R \boldsymbol{\nabla}_{s} \cdot \mathbf{u}(a, \theta, \varphi) \\
{[\boldsymbol{R} \cdot \boldsymbol{\nabla} \times \mathbf{u}]_{R=a}} & =\boldsymbol{R} \cdot \boldsymbol{\nabla}_{s} \times \mathbf{u}(a, \theta, \varphi)
\end{array}
$$

where $\boldsymbol{\nabla}_{s} \cdot \mathbf{u}$ and $\boldsymbol{\nabla}_{s} \times \mathbf{u}$ denote the tangential divergence and curl along the surface $^{13}$. The terms on the right contain only surface derivatives of the surface displacement. If this surface displacement is known, these surface derivatives can be computed from the surface displacement using only derivatives with respect to the angular variables $\theta, \varphi$ as show the following relationships:

$$
\begin{aligned}
\boldsymbol{\nabla}_{s} \cdot \mathbf{u}= & 2 \frac{u_{R}}{R}+\frac{1}{R} \frac{\partial u_{\varphi}}{\partial \varphi} \\
& +\frac{u_{\varphi}}{R} \cot \varphi+\frac{1}{R \sin \varphi} \frac{\partial u_{\theta}}{\partial \theta}
\end{aligned}
$$

and

$$
\left[\boldsymbol{\nabla}_{s} \times \mathbf{u}\right]=\frac{1}{R^{2} \sin \varphi}\left[\frac{\partial}{\partial \varphi}\left(u_{\theta} R \sin \varphi\right)-\frac{\partial}{\partial \theta}\left(R u_{\varphi}\right)\right]
$$


From another hand, the left terms of (22) can be computed using the harmonic potentials. If one calls $f_{(n)}$ the contribution of the potentials of order $n$ to $f$, one obtains the following expressions in terms of harmonic potentials:

$$
\begin{gathered}
A_{n}=u_{R(n)}=\mathbf{e}_{R} \cdot \mathbf{u}_{(n)}=\left(n-M_{n}\right) R \psi_{n}+n \frac{1}{R} \phi_{n} \\
B_{n}=\boldsymbol{R} \cdot \boldsymbol{\nabla} u_{R(n)}-R \boldsymbol{\nabla} \cdot \boldsymbol{u}_{(n)}= \\
+\frac{n \psi_{n}\left[n(n-1)+2 M_{n}\right]}{R} \\
+\frac{n-1) \phi_{n}}{R} \\
C_{n}=\boldsymbol{R} \cdot \boldsymbol{\nabla} \times \boldsymbol{u}_{(n)}=n(n+1) \chi_{n}
\end{gathered}
$$

As defined before, $\psi_{n}, \phi_{n}, \chi_{n}$ are "solid" spherical harmonics of order $n$. As such, they are given by $r^{n} Y_{m n}(\theta, \varphi)$ where $Y_{m n}$ are "surface" spherical harmonics (more precisely, these functions are surface eigenfunctions for spherical boundaries coming from the separation of variables applied to Laplace equation in spherical coordinates). These "surface" harmonics are given by $Y_{m n}=\cos (m \theta) P_{n}^{m}(\cos \varphi)$ or $Y_{m n}=\sin (m \theta) P_{n}^{m}(\cos \varphi)$, where $P_{n}^{m}$ are the Legendre functions ${ }^{24}$.

For the problem under consideration, the internal sphere is subjected to a uniform displacement along axis $x_{3}$. Then, the displacement field does not depend on $\theta$, leading to $m=0$. Therefore, the only surface spherical harmonics in the expansion are

$$
Y_{m n}=P_{n}^{0}(\cos \varphi)
$$


These surface spherical harmonics are not independent and comply with $Y_{m n}=Y_{m ;-n-1}$. The surface spherical harmonics with a positive value of $n$ constitute a complete orthogonal basis of functions defined on the surface of the sphere.

Finally, the right terms of equation (22), that are known if the surface displacement is known on the sphere of radius $a$, can be expanded along the orthogonal basis of "surface" spherical harmonics as:

$$
\begin{aligned}
& \mathbf{e}_{R} \cdot \mathbf{u}=\sum_{n=0}^{\infty} U_{a ; n}(\theta, \varphi) \\
& R \boldsymbol{\nabla}_{s} \cdot \mathbf{u}=\sum_{n=0}^{\infty} V_{a ; n}(\theta, \varphi) \\
& \mathbf{R} \cdot \nabla_{s} \otimes \mathbf{u}=\sum_{n=0}^{\infty} W_{a ; n}(\theta, \varphi)
\end{aligned}
$$

where $U_{a ; n}, V_{a ; n}, W_{a ; n}$ are surface harmonics that are the projections of the given surface displacement on the basis of surface harmonics of $n^{\text {th }}$ order, with a similar relationship for $R=b$.

Using these surface harmonics, the boundary conditions can be written as:

$$
\begin{aligned}
& A_{n}(a)+A_{-n-1}(a)=U_{a ; n} \\
& B_{n}(a)+B_{-n-1}(a)=V_{a ; n} \\
& C_{n}(a)+C_{-n-1}(a)=W_{a ; n}
\end{aligned}
$$

with similar relationships at the surface of the sphere of radius $b$.

Let us now consider the displacement $\mathbf{U}=U \mathbf{e}_{3}$ of the inner sphere of radius $b$. One has: 


$$
\mathbf{e}_{R} \cdot \mathbf{U}=U_{r}=U \cos \varphi=U P_{1}^{0}
$$

This problem corresponds to $n=1$ and the boundary conditions coming from all surface terms lead to $U_{a ; 1}=0, U_{b ; 1}=U \cos \varphi$, all terms in $V$ and $W$ being null. In absence of coupling between $\chi_{n}$ and other potentials, this leads to $\chi_{n}=0, \forall n$. Setting these boundary conditions makes it possible to find the displacement and stress fields inside the coating. Computing the tractions along the boundary of the coating and integrating these tractions leads to the resultant $F$ applied to the coating for a given displacement $U$ of the inner inclusion. The algebra describing these operations is reported in Appendix.

\section{Eigenfrequency of the composite inclusion}

Finally, the radial frequency at resonance $\omega_{r}$ is given for a mass $m^{(3)}$ of the inner inclusion by:

$$
\begin{aligned}
\omega_{r}^{2}= & \frac{F}{U m^{(3)}} \\
= & \frac{\mu}{\rho^{(3)} a^{2}} \cdot \frac{36}{H}(2-3 \nu)(1-\nu) \\
& \quad \cdot \frac{1+\alpha+\alpha^{2}+\alpha^{3}+\alpha^{4}}{\alpha^{2}}=\frac{\mu}{\rho^{(3)} a^{2}} \omega_{d}^{2}
\end{aligned}
$$

with 


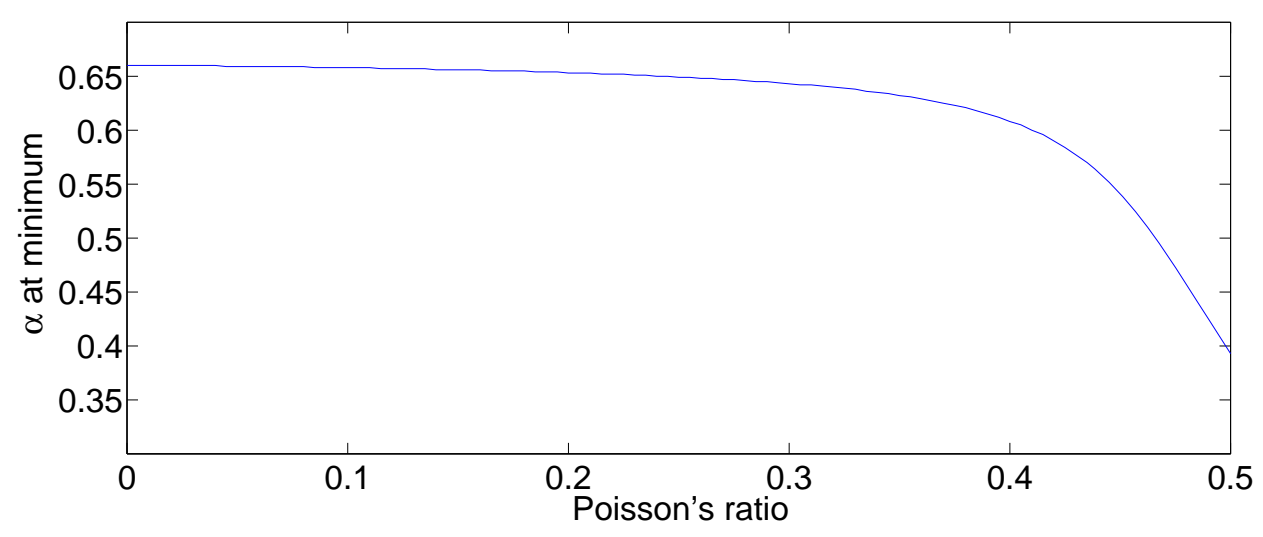

Figure 7: Ratio of the radii of the spherical composite inclusion for the minimum of resonance frequency as a function of Poisson's ratio.

$$
\begin{aligned}
H & =(1-\alpha)\left[-2(3 \nu-2)(6 \nu-5)\left(1+\alpha^{4}\right)\right. \\
& +\left(-36 \nu^{2}+54 \nu-\frac{35}{2}\right)\left(\alpha+\alpha^{3}\right) \\
& \left.+3\left(-12 \nu^{2}+18 \nu-5\right) \alpha^{2}\right]
\end{aligned}
$$

This expression displays again a non-dimensional frequency $\omega_{d}$ depending only on the geometry and on the Poisson's ratio. As for cylindrical fibers, studying for $\nu$ fixed the variation of $\omega_{d}^{2}$ as a function of $\alpha$ shows that $\omega_{d}^{2}$ has only one minimum and that an "optimal" resonance frequency does appear for this minimum when $\alpha=\alpha_{\min }$

Fig. 7 shows the variation of $\alpha_{\min }$ as a function of the Poisson's ratio. Once again, the curve displays a decrease of $\alpha_{\min }$ when the Poisson's ratio increases. Finally Fig.8 displays the "optimal" value of non-dimensional frequency as a function of the Poisson's ratio $\nu$, 


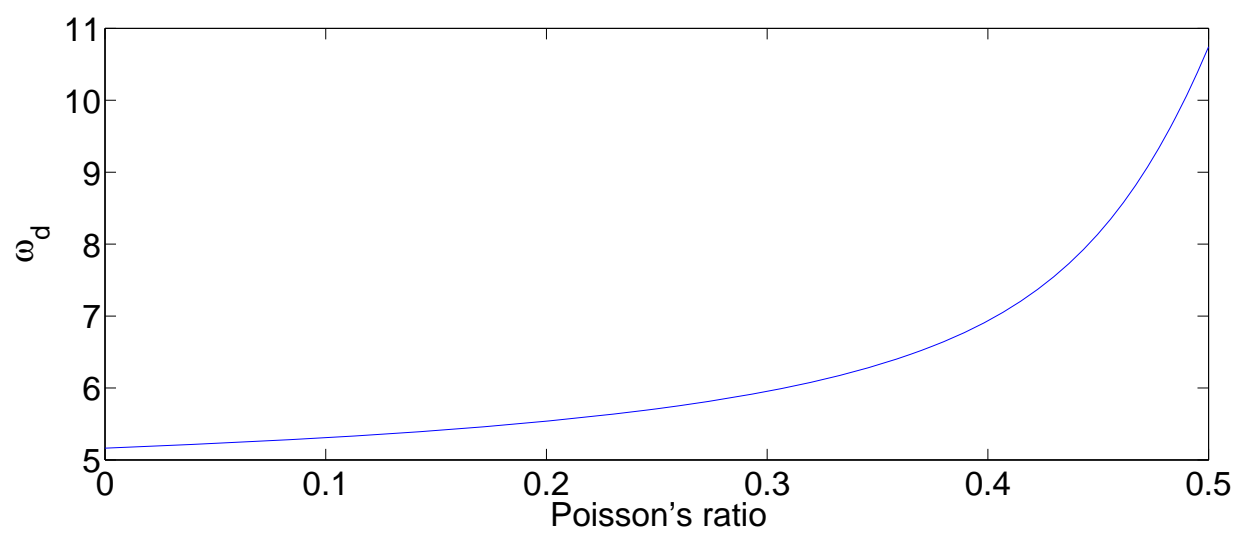

Figure 8: Minimum of the non-dimensional radial frequency at resonance of a spherical composite inclusion as a function of Poisson's ratio.

showing again that $\omega_{d}$ increases with $\nu$. As an example of application, let us now consider the case of a material containing composite spherical inclusions of the same materials as for the material containing composite fibers in the previous section. Fig.9 displays the variation of resonance frequency for "optimal " geometries as a function of the external radius of composite inclusions, showing that the resonance frequency lies between $5 \mathrm{kHz}$ and $5 \mathrm{MHz}$ for an external diameter of composite inclusion varying between $1 \mathrm{~mm}$ and $1 \mu m$.

Finally, Fig.10 displays the ratio between dynamic mass density and static mass density for the previously defined composite material containing equal parts (in volume) of matrix and (composite) inclusions. Within the composite inclusions, the ratio of inner 


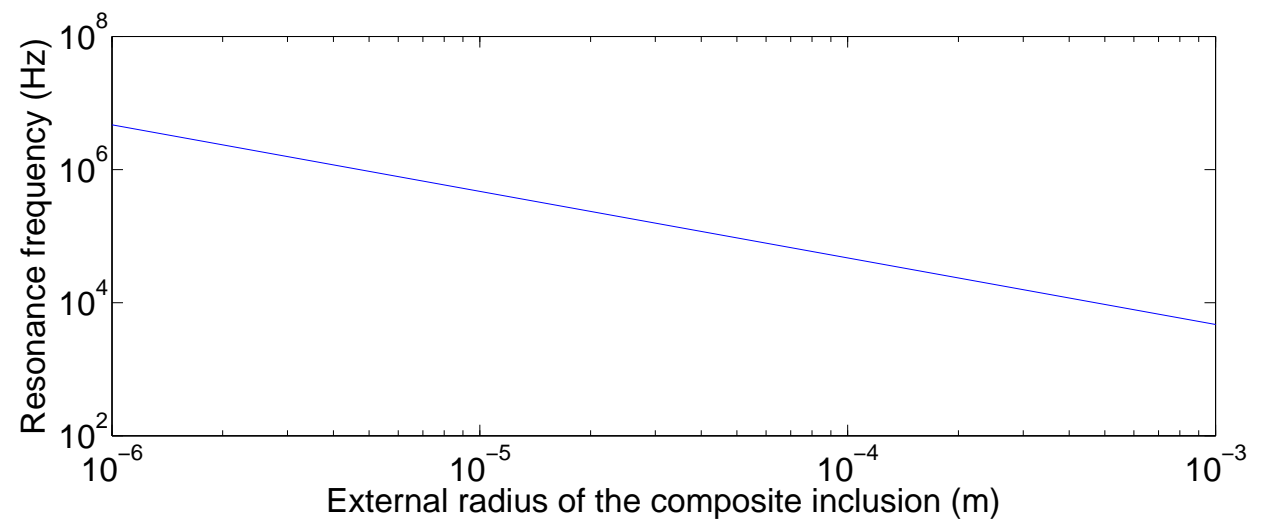

Figure 9: Resonance frequency $(\mathrm{Hz})$ of a material with given physical properties containing spherical inclusions as a function of the outer diameter.

inclusion and coating are those coming from the "optimal" ratio such as defined before. The choice of the window hides the fact that the dynamic mass density tends towards the static mass density at low frequencies. It can be seen that the window corresponding to a negative value of the mass density extends between 4.7 and $5.3 \mathrm{kHz}$. The minimal frequency value of the window corresponds practically to the resonance frequency. The maximal value of the window corresponds to the frequency such as the negative part of the dynamic mass density equilibrates the static mass density. Obviously, if the minimal frequency value depends only on the resonance frequency, the maximal value of the window (and its width) depends also on the ratio between the dynamic and static parts of the mass density: it increases with the proportion of composite inclusions.

\section{CONCLUSION}



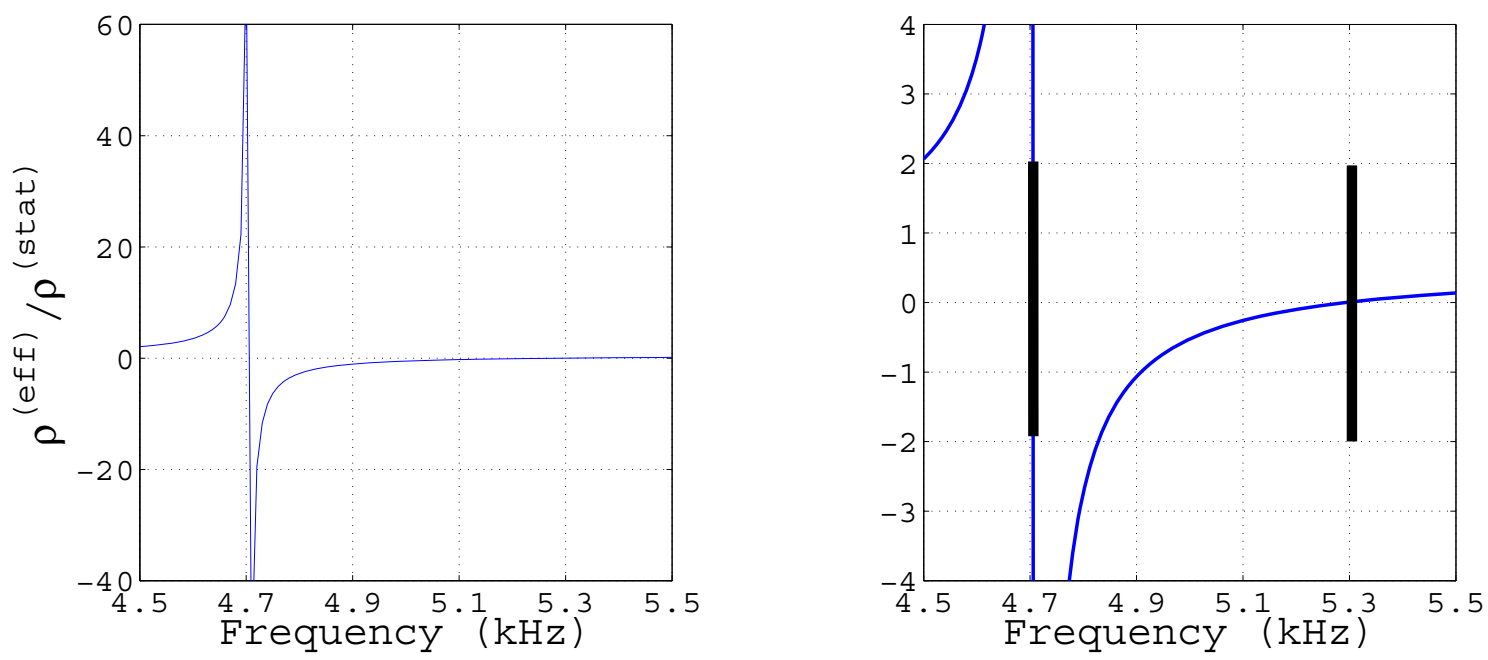

Figure 10: Relative dynamic mass density $\rho^{(e f f)} / \rho^{(\text {stat })}$ as a function of frequency (kHz). Right: zoom to show the range where $\rho_{\text {eff }}$ is negative, denoted by thick vertical bars.

In this paper, the basic results obtained from the homogenization theory using asymptotic expansions have been summarized for composite elastic metamaterials with composite inclusions displaying inner resonance. These results show that the dynamic behaviour of the composite displays a frequency-dependent and tensorial mass density. This mass density can be computed knowing the resonance frequencies of the inclusions for the condition of null displacement at the surface of the composite inclusion. This greatly simplifies the computation of the dynamic mass density. In addition, it shows that all resonators are independent. This corresponds to the idea of inner resonance.

The resonance frequencies of these resonators have been computed successfully in the 
case of cylindrical or spherical composite inclusions. They have been obtained in the form of simple analytical expressions and the results show that these resonance frequencies are minimal for a specific," optimal", geometry of the composite inclusion. These results lead to closed form expressions of the resonance frequencies and of the dynamic mass density corresponding to cylindrical and spherical composite inclusions acting as "spring-mass" resonators.

\section{APPENDIX: DETAIL OF THE COMPUTATION OF THE STIFFNESS OF SPHERICAL COMPOSITE INCLUSIONS}

From equations $(25,26,30)$ and the first two equations of $(31)$, the displacement conditions on the boundaries of the coating lead to the following system of equations for the unknown values of potentials $\phi_{1}, \psi_{1}, \phi_{-2}, \psi_{-2}$ on the boundaries:

$$
\begin{aligned}
& L_{1} a \psi_{1}(a)+\frac{1}{a} \phi_{1}(a)-N_{1} a \psi_{-2}(a)-\frac{2}{a} \phi_{-2}(a)=0 \\
& 2 M_{1} a \psi_{1}(a)+a Q_{1} \psi_{-2}(a)+6 \frac{\phi_{-2}(a)}{a}=0 \\
& L_{1} b \psi_{1}(b)+\frac{1}{b} \phi_{1}(b)-N_{1} b \psi_{-2}(b)-\frac{2}{b} \phi_{-2}(b)=U \cos \varphi \\
& 2 M_{1} b \psi_{1}(b)+b Q_{1} \psi_{-2}(b)+6 \frac{\phi_{-2}(b)}{b}=0
\end{aligned}
$$

where $L_{1}=1-M_{1}, N_{1}=2+M_{-2}, Q_{1}=6+2 M_{-2}$.

However, the values of potentials at $a$ and $b$ are not independent: due to the radial 
dependence of spherical harmonics, only four values of boundary potentials are independent. The system is rewritten using the values of all potentials at $R=a$. Finally, the solution of the linear system gives $\psi_{1}(a), \phi_{1}(a), \omega_{-2}(a), \phi_{-2}(a)$, leading to the potentials given by:

where :

$$
\begin{aligned}
& \psi_{1}=A_{1} R \cos \varphi \\
& \phi_{1}=B_{1} R \cos \varphi \\
& \psi_{-2}=A_{-2} \frac{\cos \varphi}{R^{2}} \\
& \phi_{-2}=B_{-2} \frac{\cos \varphi}{R^{2}}
\end{aligned}
$$

$$
\begin{aligned}
& A_{1}=\frac{1}{a^{2}} \frac{3 \alpha S_{1}(\alpha+1)}{D L_{1}} U \\
& B_{1}=\alpha \frac{T_{1} \alpha^{2}\left(S_{1}-3\right)\left(1-\alpha^{3}\right)-3\left(S_{1}+T_{1}\right)\left(1-\alpha^{2}\right)}{D(1-\alpha)} U \\
& A_{-2}=\frac{-3 a \alpha T_{1}\left(1+\alpha+\alpha^{2}+\alpha^{3}+\alpha^{4}\right)}{D N_{1}} U \\
& B_{-2}=\frac{a^{3} T_{1} S_{1} \alpha^{3}\left(1+\alpha+\alpha^{2}\right)}{2 D} U
\end{aligned}
$$

with

$$
\begin{aligned}
D= & (1-\alpha)\left[T_{1}\left(3-S_{1}\right)\left(1+\alpha^{4}\right)\right. \\
& +\left(-3 S_{1}-2 T_{1} S_{1}+3 T_{1}\right)\left(\alpha+\alpha^{3}\right) \\
& \left.+3\left(T_{1}-T_{1} S_{1}-2 S_{1}\right) \alpha^{2}\right]
\end{aligned}
$$

and where $\alpha$ is the ratio of radii $b / a . S_{1}, T_{1}$ are functions of Poisson's ratio through 
$M_{1}, M_{-2}$ given by

$$
\begin{aligned}
T_{1} & =\frac{2 M_{1}}{1-M_{1}} \\
S_{1} & =\frac{6+2 M_{-2}}{2+M_{-2}}
\end{aligned}
$$

The general expression for the traction vector $\mathbf{f}$ around the sphere of radius $R$ as a function of the potentials is given ${ }^{31}$ by:

$$
\begin{aligned}
\frac{\mathbf{f}}{\mu}= & \sum_{n} \gamma_{n} \psi_{n} \mathbf{e}_{R}+\beta_{n} R \nabla \psi_{n} \\
& +\frac{2(n-1)}{R} \nabla \phi_{n}+(n-1) \nabla \chi_{n} \times \mathbf{e}_{R}
\end{aligned}
$$

where $\gamma_{n}, \beta_{n}$ are:

$$
\begin{aligned}
& \beta_{n}=2 n-M_{n} \\
& \gamma_{n}=2 n-(2+n) M_{n}+\frac{2 \nu}{1-2 \nu}\left(2 n-M_{n}(n+3)\right)
\end{aligned}
$$

Accounting for the potentials appearing in this particular case of boundary condition, only the potentials for $n=1, n=-2$ do appear in the solution. From another hand, the resultant of the tractions over the boundary of the coating is oriented along $x_{3}$ direction and only the projection $f_{3}$ of the traction vector along $x_{3}$ contributes to the resultant, with

$$
\begin{aligned}
\frac{f_{3}}{\mu} & =\left(\gamma_{1} \psi_{1}+\gamma_{-2} \psi_{-2}\right) \cos \varphi \\
& +R\left(\beta_{1} \nabla \psi_{1}+\beta_{-2} \nabla \psi_{-2}\right) \cdot \mathbf{e}_{3}-\frac{6}{R} \nabla \phi_{-2} \cdot \mathbf{e}_{3}
\end{aligned}
$$


The resultant is finally given by:

$$
\begin{aligned}
F & =\int_{0}^{\pi} \int_{0}^{2 \pi} f_{3} R^{2} \sin \varphi d \theta d \varphi \\
& =2 \pi R^{2} \int_{0}^{\pi} f_{3} \sin \varphi d \varphi
\end{aligned}
$$

The integration over the inner spherical boundary leads to:

$$
\frac{F}{2 \pi b^{2} \mu}=-\frac{2 U R_{1} \gamma_{-2}}{\alpha a D N_{1}}\left(1+\alpha+\alpha^{2}+\alpha^{3}+\alpha^{4}\right)
$$

that gives the stiffness of the composite inclusion.

\section{I. * REFERENCES}

1. W. Akl and A. Esabbagh and A. Baz, "Acoustic metamaterials with circular sector cavities and programmable densities", J. Acoust. Soc. Am. 132(4), 2857-2865 (2012).

2. J.L. Auriault and G. Bonnet, "Dynamique des composites élastiques périodiques(Dynamics of elastic periodic composites)", Arch. Mech. 37(4), 269-284 (1985).

3. J.L. Auriault, "Acoustics of heterogeneous media: macroscopic behavior by homogenization", Current Topics Acoust. Res. 1, 63-90 (1994).

4. J.L. Auriault and C. Boutin, "Long wavelength inner-resonance cut-off frequencies in elastic composite materials", Int. J. Solids Struct. 49(23-24), 3269-3281 (2012). 
5. A. Avila and G. Griso and B. Miara, "Phononic bandgaps in linearized elasticity", Comptes Rendus Acad. Sci. I. 340(12), 933-938 (2005).

6. N.O. Babych and I.V. Kamotski and V.P. Smyshlyaev, "Homogenization of spectral problems in bounded domains with doubly high contrasts", Networks and Heterogeneous Media. 3(3), 413-436 (2008).

7. N.S. Bakhvalov and G.P. Panasenko, Homogenization averaging process in periodic media. Mathematical problems in the mechanics of composite materials (Kluwer, Dordrecht, 1989), pp.1-366.

8. A. Baz, "An active acoustic metamaterial with tunable effective density", ASME J. of Vibration and Acoustics. 132(4), 0410111-0410119 (2010).

9. A. Bensoussan and J.L. Lions and G. Papanicolaou, Asymptotic analysis for periodic structures (North Holland, Amsterdam, 1978), pp.1-392.

10. J. Carbonell and F. Cervera and J. Sanchez-Dehesa and J. Ariaga and L. Gumen and A. Krokhin, "Homogenization of two-dimensional anisotropic dissipative photonic crystal", Appl. Phys. Lett. 97(4), 231122 (2010).

11. R. Gracia-Salgado and D.Torrent and J. Sanchez-Dehesa, "Double-negative acoustic 
metamaterials based on quasi-two-dimensional fluid-like shells, New J. of Phys. $\mathbf{1 4}, 103052(2012)$

12. W.A. Gross, "The second fundamental problem of elasticity applied to a plane circular ring", Z. Ang. Math. Phys. 8,71-73 (1957).

13. M.E. Gurtin and A.I. Murdoch, "Continuum theory of elastic material surfaces", Arch. Rat. Mech. Anal. 57(4),291-323 (1975).

14. J. Happel and H. Brenner, Low Reynolds Number Hydrodynamics (Nordhoff, Leiden,1983), 1-553.

15. H.H. Huang and C.T. Sun, "Locally resonant acoustic metamaterials with 2D anisotropic effective mass density", Phil. Mag. 91(6),981-996 (2011).

16. H.H. Huang and C.T. Sun, "Anomalous wave propagation in a one-dimensional acoustic metamaterial having simultaneously negative mass density and Young's modulus", J. Acous. Soc. Am. 132(4),2887-2895 (2012).

17. A.A. Krokhin and J. Arriaga and L.N. Gumen, "Speed of sound in periodic elastic composites", Phys. Rev. Lett. 91(26),2643021-2643024 (2003).

18. S.H. Lee and C.M. Park and Y.M. Seo and Z.G. Wang and C.K. Kim, "Acoustic metamaterial with negative density", Phys. Lett. A. 373(48),4464-4469 (2009). 
19. Z. Liu and X. Zhang and Y. Mao and Y.Y. Zhu and Z. Yang and C.T. Chan and P. Sheng, "Locally resonant materials", Science 289,1734-1736 (2000).

20. A.E.H. Love, A treatise on the mathematical theory of elasticity (Dover Publ.,N.Y.,1944), pp. 249-251.

21. A.I. Lurie, Three-dimensional problems of the theory of elasticity (Interscience, New York,1964),pp. 462-472.

22. G.W. Milton, "New metamaterials with macroscopic behavior outside that of continuum elastodynamics", New J. Phys. 9,359 (2007).

23. G.W. Milton and J.R. Willis, "On modifications of Newton's second law and linear continuum elastodynamics", Proc. Roy. Soc. A. 463,855-880 (2007).

24. P.M. Morse and H. Feshbach, Methods of theoretical physics. (McGraw-Hill, N.Y.,1953),p. 1264.

25. N.I. Muskhelishvili, Some basic problems of the mathematical theory of elasticity (Martinus-Nijhoff, Dordrecht,1977),pp.230-249.

26. C.J. Naify and C.M. Chang and G. Mcknight and S.R. Nutt, "Scaling of membrane-type locally resonant acoustic metamaterial arrays", J. Acoust. Soc. Am. 132(4),2784-2792 (2012). 
27. S. Nemat-Nasser and J.R. Willis and A. Srivastava and A.V. Amirkhizi, "Homogenization of periodic elastic composites and locally resonant materials", Phys. Rev. B 83,104103 (2011).

28. A.N. Morris and A.L. Shuvalov and A.A. Kutsenko, "Analytical formulation of three-dimensional dynamic homogenization ”, Proc. R. Soc. A 468,1629-1651 (2012).

29. J. Park and B. Park and D. Kim and J. Park, "Determination of effective mass density and modulus for resonant metamaterials", J. Acoust. Soc. Am. 132(4),2793-2799 (2012).

30. W.J. Parnell and I.D. Abrahams, "Dynamic homogenization in periodic fibre reinforced media. Quasi-static limit for SH waves", Wave Motion 43,474-498 (2006).

31. M. Rahman and T. Michelitsch, "A general procedure for solving boundary-value problems of elastostatics for a spherical geometry based on Love's approach", Quart. J. Mech. Appl. Mech. 60(2),139-160 (2007).

32. E. Shamonina and L. Solymar, "Metamaterials: How the subject started", Metamaterials. 1,12-18 (2007).

33. P. Sheng and X.X. Zhang and Z. Liu and C.T. Chan, "Locally resonant sonic materials", Metamaterials. 338,201-205 (2003). 
34. J. Soubestre and C. Boutin, "Non-local dynamic behavior of linear fiber reinforced materials", Mech. Mat. 55,16-32 (2012).

35. J.O. Vasseur and P.A. Deymier and G. Prantziskonis and G. Hong, "Experimental evidence for the existence of absolute acoustic band gaps in two-dimensional periodic composite media", J. Phys.: Condens. Mater. 10,6051-6064 (1998).

36. V.G. Veselago, "The electrodynamics of substances with simultaneously negative values of $\epsilon$ and $\mu "$, Sov. Phys. Usp. 10,509-514 (1968).

37. Z. Yang and J. Mei and M. Yang and N. H. Chan and P. Sheng, "Membrane-Type Acoustic Metamaterial with Negative Dynamic Mass", Phys. Rev. Lett. 101,204301 (2008)

38. S. Yao and X. Zhou and G. Hu, "Experimental study on negative effective mass in a 1D mass-spring system", New J. Phys. 10,043020 (2008). 